\title{
TRANSPLANTE HEPÁTICO NA INSUFICIÊNCIA HEPÁTICA AGUDA GRAVE INDUZIDA POR IMATINIBE
}

\author{
Liver transplantation due to Imatinib induced severe acute liver failure
}

\author{
Danilo Nakaya Alvarenga de Rezende1, Natalia Campregher Confuorto Romano1, Gabriela Tomaz Martinho1, \\ Pedro de Souza Lucarelli Antunes ${ }^{1}$, Talita Di Santi', Marcelo Callado Fantauzzi ${ }^{1}$, Bruno Vaz Kerges Bueno ${ }^{2}$ e André Ibrahim David ${ }^{3}$
}

\section{RESUMO}

AMM, sexo feminino, 40 anos, portadora de leucemia mieloide crônica (LMC), em uso de Imanitibe $400 \mathrm{mg} / \mathrm{dia}$ desde o diagnóstico da doença, em 13/06/2016; foi internada no dia 21/01/2018 para investigação, com história de icterícia, náuseas e vômitos em grande quantidade há uma semana da data da internação. Após seis dias, a paciente foi diagnosticada com insuficiência hepática aguda grave, apresentava-se ictérica 4+/4+ e com bilirrubina de 5,41 g/dL, albumina de 2,8, INR de 4,1, ascite moderada, creatina sérica de $0,7 \mathrm{mg} / \mathrm{dL}$. O transplante foi realizado 13 dias após a internação com fígado de doador falecido, com morte encefálica. Procedimento foi realizado sem intercorrências. Imatinibe é um inibidor seletivo da BCR-ABL tirosina quinase, uma enzima que tem atividade na Leucemia Mieloide Crônica (LMC) e tumores estromais gastrointestinais. A droga é metabolizada no fígado pelo sistema de enzimas CYP3A4, e gera muitos metabólitos ativos. Reportamos um caso de uma mulher de 40 anos, em uso de Imatinibe por um ano e sete meses até o reconhecimento de sua hepatoxicidade. Na literatura, recomenda-se que sejam feitos testes de função hepática antes do início do tratamento de LMC, e que tais parâmetros sejam monitorizados mensalmente ou segundo recomendações clínicas. Além disso, é possível observar que há boa recuperação da hepatotoxicidade de alguns pacientes apenas com a parada da droga, se as alterações hepáticas forem diagnosticadas a tempo. $O$ manejo mais eficiente da LMC após o transplante ainda não foi estabelecido. No caso reportado, o uso de PRISMA antes, durante e após o transplante pode ter sido responsável pelo bom prognóstico.

Descritores: Imatinibe, Transplante Hepático, Insuficiência Hepática.

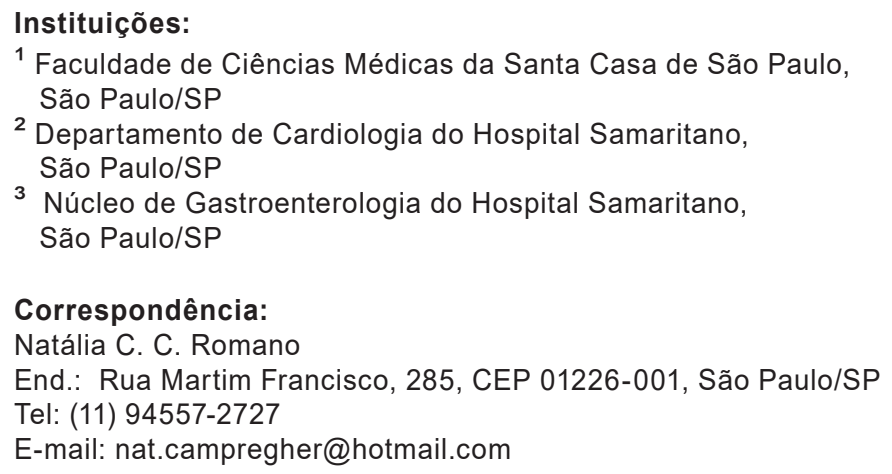

\section{CASO}

AMM, sexo feminino, 40 anos, portadora de leucemia mieloide crônica (LMC), em uso de Imanitibe $400 \mathrm{mg} /$ dia, desde o diagnóstico da doença em 13/06/2016, e Hemirtarato de Zolpidem, foi internada no dia 21/01/2018 para investigação, com história de icterícia, náuseas e vômitos em grande quantidade, há uma semana. Paciente apresentava infecção prévia por Cocksakievirus há quatro meses; realizou cirurgia bariátrica em 2008; negava uso de Paracetamol. 
Ao exame físico, encontrava-se lentificada, letárgica, ictérica $(1+/ 4+)$, desidratada $(2+/ 4+)$. Apresentou bulhas rítmicas hipofonéticas, murmúrios vesiculares presentes e diminuídos em base, abdome globoso com distensão moderada, ascite, fígado palpável há $2 \mathrm{~cm}$ do rebordo costal, perfusão regular e discreto Flapping. $\mathrm{Na}$ admissão, o uso de Imatinibe foi interrompido.

Foram solicitados exames complementares que apresentaram os seguintes resultados: Amônia de $200 \mathrm{mg} / \mathrm{dL}$ (parâmetro normal: $<100 \mathrm{mg} / \mathrm{dL}$ ), INR (International Normalized Ratio) de 3,2, AST de 1420 (parâmetro normal: 5-40 unidades/L), ALT de 1297 (parâmetro normal: 7-56 unidades/L), plaquetas de $84.000 \mu \mathrm{l}$ (parâmetro normal: 150.000-400.000 $\mu \mathrm{l}$ ), fibrinogênio de 170 mg/dL (parâmetro normal: 195 a 365 $\mathrm{mg} / \mathrm{dl}$ ), e fator $\mathrm{V}$ de $29 \%$. Sorologias negativas para hepatites A, B e C. A ultrassonografia de abdome total apresentou fígado com tamanho reduzido, porém com lobo direito proeminente, contornos serrilhados e textura difusamente heterogênea, sugestivo de hepatopatia parenquimatosa grave.

Em ultrassom prévio, de outubro de 2017, o fígado apresentava-se normal, sugerindo acometimento hepático agudo.

Após seis dias de internação, a paciente foi diagnosticada com insuficiência hepática aguda grave, apresentandose ictérica 4+/4+ e com bilirrubina de $5,41 \mathrm{~g} / \mathrm{dL}$, albumina de 2,8 , INR de 4,1 , ascite moderada (mostrada na imagem de tomografia computadorizada das figuras 1 e 2), creatina sérica de $0,7 \mathrm{mg} / \mathrm{dL}$, sem déficits neurológicos motores e Flapping positivo e rebaixamento do nível de consciência. Paciente prontamente foi submetida a plasmaferese (PRISMA) e, por preencher critérios de Clichy - fator $V<30 \%$ em pacientes com mais de 30 anos - foi indicado o transplante hepático.

Transplante foi realizado 13 dias após a internação, com fígado de doador falecido, com morte encefálica, com tempo de isquemia morna de 33 minutos e tempo de isquemia total (fria + morna) de 7 horas e 13 minutos. Procedimento foi realizado sem intercorrências.

Na patologia cirúrgica, encontrou-se necrose confluente em todos os segmentos hepáticos e nódulo de hepatócitos remanescentes nos segmentos III, IV e VIII. Após cirurgia, continuou com o uso do PRISMA e a paciente apresentava Meld de 37. Após 20 dias da cirurgia, foi necessária uma nova abordagem para drenagem e limpeza da infecção de subcutâneo com secreção e controle do sangramento com aspiração de ascite hemática.

Paciente evoluiu estável e teve alta hospitalar após 34 dias.

\section{DISCUSSÃO}

Imatinibe é um inibidor seletivo da BCR-ABL tirosina quinase, uma enzima que tem atividade na Leucemia Mieloide Crônica (LMC) e tumores estromais gastrointestinais. A droga é metabolizada no fígado pelo sistema de enzimas CYP3A4, e gera muitos metabólitos ativos. ${ }^{1}$ Apesar de amplamente utilizado, apenas $5 \%$ dos pacientes na fase crônica de LMC recebendo Imatinibe necessitaram interromper o tratamento devido aos efeitos adversos. ${ }^{2}$ Casos de hepatotoxicidade fatal e severa foram descritos.

Reportamos um caso de uma mulher de 40 anos, em uso de Imatinibe por um ano e sete meses até o reconhecimento de sua hepatoxicidade. Na literatura, entretanto, encontramos um intervalo de duas semanas a dois anos para a evolução do quadro em nove casos relatados. $^{3}$ No caso, a paciente apresentou-se, no momento da insuficiência hepática fulminante, com ictérica 4+/4+, bilirrubina de 5,41 $\mathrm{g} / \mathrm{dL}$, albumina de 2,8 , INR de 4,1 , ascite moderada, creatina sérica de $0,7 \mathrm{mg} / \mathrm{dL}$, sem déficits neurológicos motores e Flapping positivo. Dentre os sintomas descritos nos casos relatados, os únicos não encontrados no nosso caso foram febre, náusea, astenia.

Há casos descritos relacionados ao consumo de álcool e/ou Paracetamol (Acetaminofeno); E. Ridruejo et al relatam consumo de Paracetamol 500-1000 mg/ dia relacionado aos sintomas. ${ }^{4}$ No caso reportado, a paciente não utilizava outras medicações hepatotóxicas, de modo a levar a hipótese de hepatotoxicidade relacionado ao uso de Imatinibe, contudo não existe na literatura descrição dos mecanismos responsáveis pela lesão. Além disso, as lesões encontradas na biópsia de fígado não são compatíveis com as causadas pelo Paracetamol.

A paciente fazia uso de Zolpidem; os efeitos colaterais do uso de Zolpidem são relacionados à sua propriedade agonista gabaérigica, sem relação aos efeitos de hepatotoxicidade descritos na literatura. ${ }^{5}$

$\mathrm{Na}$ tabela, há uma comparação de relatos de casos reportados previamente (fatais e não fatais) de insuficiência hepática em pacientes que faziam tratamento com Imatinibe. Nota-se que, na maioria dos casos, assim como neste, os pacientes eram portadores de LMC. No entanto, poucos pacientes fizeram o tratamento com Imatinibe por mais de 77 semanas. Além disso, é possível observar que há boa recuperação de alguns pacientes apenas com a parada da droga e, em três dos casos, incluindo este, o transplante foi realizado com sucesso. 
Danilo Nakaya Alvarenga de Rezende, Natalia Campregher Confuorto Romano, Gabriela Tomaz Martinho, Pedro de Souza Lucarelli Antunes, Talita Di Santi, Marcelo Callado Fantauzzi, Bruno Vaz Kerges Bueno e André Ibrahim David

Neste trabalho, temos, portanto, dez casos relatados, sendo que a maioria sobreviveu com ou sem transplante.
Três casos foram a óbito, sendo dois antes do transplante e um após, como mostra a tabela 1.

Tabela 1 - Levantamento bibliográfico das complicações hepáticas do Imatinib

\begin{tabular}{|c|c|c|c|c|}
\hline Referências & Diagnóstico & $\begin{array}{l}\text { Tempo para } \\
\text { disfunção } \\
\text { hepática }\end{array}$ & Histologia hepática & $\begin{array}{l}\text { Resultado das } \\
\text { enzimas } \\
\text { hepáticas }\end{array}$ \\
\hline Ohyashiki et al (2002) & LMC & 12 dias & Necrose focal dos hepatócitos & Resolução após cessar a droga \\
\hline Lin et al (2003) & Policitemia vera & 7 semanas & Necrose hepática & $\begin{array}{l}\text { Falência hepática aguda. } \\
\text { Paciente faleceu }\end{array}$ \\
\hline James et al (2003) & LMC & 49 semanas & $\begin{array}{l}\text { Hepatite citolítica severa aguda. } \\
\text { Necrose e colestase leve }\end{array}$ & Resolução após cessar a droga \\
\hline Kikuchi et al (2004) & LMC & 36 semanas & Necrose hepática & Resolução após cessar a droga \\
\hline Ayoub et al (2004) & LMC & 96 semanas & $\begin{array}{l}\text { Inflamação portal e lobular. Necrose lobular } \\
\text { multifocal }\end{array}$ & Resolução após cessar a droga \\
\hline Cross et al (2007) & LMC & 77 semanas & $\begin{array}{l}\text { Necrose grave com queda de células } \\
\text { confluentes multilobulares. Colapso de } \\
\text { reticulina, regeneração multinodular }\end{array}$ & $\begin{array}{l}\text { Paciente faleceu após } 10 \text { dias } \\
\text { do transplante }\end{array}$ \\
\hline Ridruejo et al (2007) & LMC & 22 semanas & Não disponínel & $\begin{array}{l}\text { Hepato necrose } \\
\text { Falência hepática aguda } \\
\text { Paciente faleceu }\end{array}$ \\
\hline Este Caso & LMC & 77 semanas & $\begin{array}{l}\text { Necrose confluente em todos os segmentos } \\
\text { hepáticos sem indícios de malignidade }\end{array}$ & $\begin{array}{l}\text { Resolução após parada da droga } \\
\text { e transplante hepático }\end{array}$ \\
\hline Harding et al (2016) & LMC & 20 semanas & $\begin{array}{l}\text { Necrose submaciça aguda com inflamação leve } \\
\text { a moderada do trato portal, incluindo osinófilos } \\
\text { ocasionais, mas sem plasmócitos }\end{array}$ & $\begin{array}{l}\text { Resolução após parada da droga } \\
\text { e transplante hepático }\end{array}$ \\
\hline Perini et al (2009) & LMC & 72 semanas & $\begin{array}{l}\text { Necrose Panacinar em } 85 \% \text { do fígado, com } \\
\text { colestase extensa. } \\
\text { Doença ductular reação }\end{array}$ & $\begin{array}{l}\text { Resolução após parada da droga } \\
\text { e transplante hepático }\end{array}$ \\
\hline
\end{tabular}

LMC: Leucemia mieloide crônica

Figuras 1 e 2 - Tomografia de abdome e pelve demonstrando grande quantidade de ascite, sem doença hepática crônica
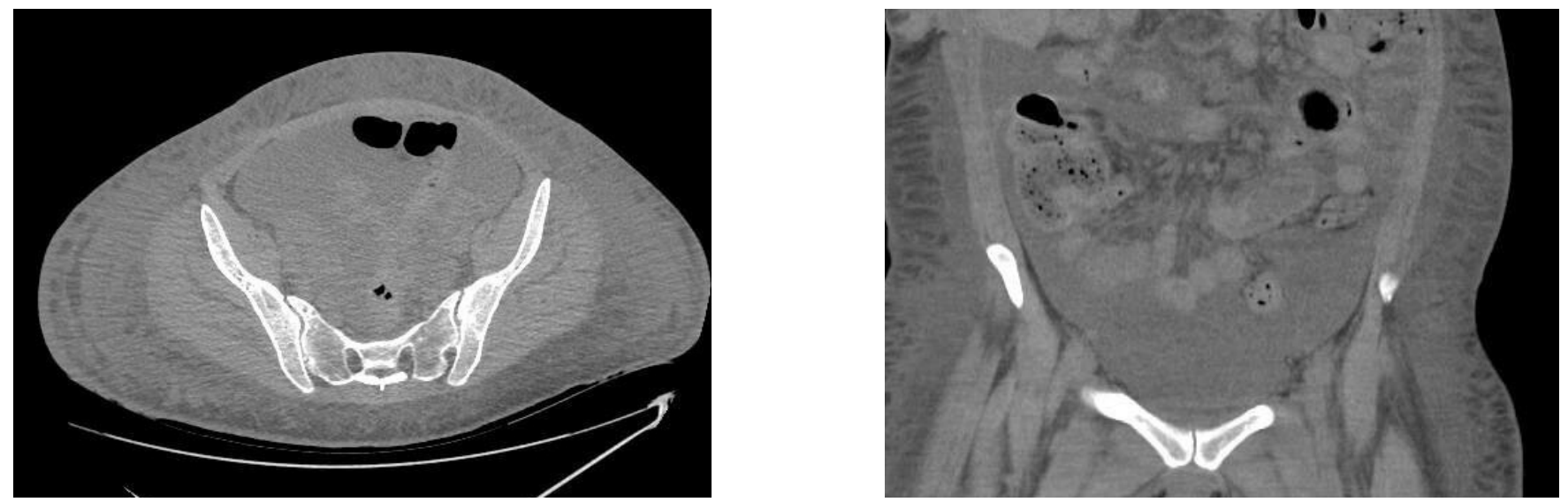
$\mathrm{Na}$ literatura, recomenda-se que sejam feitos testes de função hepática antes do início do tratamento e que tais parâmetros sejam monitorizados mensalmente ou segundo as recomendações clínicas. ${ }^{4}$

Ferrero et al reportaram que corticoesteroides em baixas a médias doses podem reverter a hepatotoxicidade induzida pelo Imatinibe. O uso de prednisona (25-37 $\mathrm{mg} / \mathrm{dia}$ ) ou metilprednisona (40 $\mathrm{mg} / \mathrm{dia}$ ) resultam na normalização dos níveis séricos das aminotransferase, em duas a quatro semanas, em todos os cinco pacientes tratados. ${ }^{6}$

Outros dois transplantes bem sucedidos para falência hepática induzida pelo uso de Imatinibe foram descritos, ${ }^{3,7}$ no entanto, o manejo mais eficiente da LMC após o transplante ainda não foi estabelecido3; no caso reportado, o uso de PRISMA antes, durante e após o transplante pode ter sido responsável pelo bom prognóstico. Não existem casos descritos com terapias semelhantes.

\section{CONCLUSÃO}

A rápida indicação e condução para o transplante, em nosso caso, foram bons preditores do prognóstico.

Há dez meses de pós-operatório, paciente com boa evolução, tendo apresentado apenas infecção intestinal por citomegalovírus (CMV) tratada com Ganciclovir.

No momento, apresenta-se em uso de imunossupressor (Tacrolimus, Everolimus e Mifortic) e Sprycel (Dasatinibe) $100 \mathrm{mg} / \mathrm{dia}$, como tratamento alternativo para LMC.

\section{ABSTRACT}

MMA, female, 40 years old, with chronic myeloid leukemia (CML), using Imanitibe $400 \mathrm{mg} /$ day since diagnosed on 06/13/2016; she was hospitalized for investigation on 21/01/2018, with history of jaundice, nausea and vomiting in large amounts one week from the date of hospitalization. After 6 days, patient was diagnosed with severe acute liver failure, 4+/4+ icteric and $5.41 \mathrm{~g} / \mathrm{dL}$ bilirubin, 2.8, INR 4.1 albumin, moderate ascites, $0.7 \mathrm{mg} / \mathrm{dL}$ serum level creatine. Transplantation was performed 13 days after hospitalization with the liver of a deceased donor who had brain death. Procedure was conducted without intercurrences. Imatinib is a selective BCR-ABL tyrosine kinase inhibito, an enzyme with activity in Chronic Myeloid Leukemia (CML) and gastrointestinal stromal tumors. The drug is metabolized in the liver by the CYP3A4 enzyme system, and generates many active metabolites. We report a case of a 40-year-old woman on imatinib for 1 year and 7 months until reckognizing her hepatoxicity. The literature recommends that liver function tests are performed prior to the beginning of the treatment, and these parameters be monthly monitored or following clinical recommendations. In addition, it is possible to note that there is a good recovery of the hepatotoxicity in some patients by only terminating the drug if liver changes are punctually diagnosed. The most efficient after transplantation CML management has not yet been set. In the reported case, the use of PRISMA before, during and after transplantation may have been responsible for the good prognosis.

Keywords: Imatinib, Liver Transplantation, Hepatic Insufficiency.

\section{AGRADECIMENTOS}

Agradecemos à Faculdade de Ciências Médicas da Santa Casa de São Paulo e ao

Hospital Samaritano Américas, pela colaboração intelectual

e auxílio técnico ao nosso trabalho. 


\section{REFERÊNCIAS}

1. Cohen M, Williams G, Johnson J, Duan J, Gobburu J, Rahman A, et al. Approval Summary for Imatinib Mesylate Capsules in the Treatment of Chronic Myelogenous Leukemia [Internet]. Clin Cancer Res, 2002;8(5):93542. Available from: http://clincancerres.aacrjournals.org/ content/8/5/935.full-text.pdf

2. Hughes TP, Hochhaus A, Branford S, Müller MC, Kaeda JS, Foroni L, et al. Long-term prognostic significance of early molecular response to imatinib in newly diagnosed chronic myeloid leukemia: an analysis from the International Randomized Study of Interferon and STI571 (IRIS) [Internet]. Blood, 2010;116(19): 3758-3765. Available from: https:// www.ncbi.nlm.nih.gov/pmc/articles/PMC3266053/

3. J. Harding D, MacQuillan G, Howman R, de Boer B, Adams L, Mitchell A. Successful Use of Dasatinib After Liver Transplantation for Imatinib-Induced Fulminant Liver Failure in Chronic Myeloid Leukemia [Internet]. Clin Oncol, 2016;34(11):e97-e98. Available from: https://ascopubs.org/ doi/pdf/10.1200/JCO.2013.50.1320

4. Nacif L, Waisberg D, Pinheiro R, Lima F, Rocha-Santos V, Andraus W, et al. Imatinib-induced fulminant liver failure in chronic myeloid leukemia: role of liver transplant and secondgeneration tyrosine kinase inhibitors: a case report [internet]. J Med Case Rep, 2018 Mar;12:63 Available from: https:// jmedicalcasereports.biomedcentral.com/track/pdf/10.1186/ s13256-018-1588-0

5. Atkin T, Comai S, Gobbi G. Drugs for Insomnia beyond Benzodiazepines: Pharmacology, Clinical Applications, and Discovery [Internet]. Pharmacol Rev, 2018 Apr;70(2)197245 Available from: http://pharmrev.aspetjournals.org/ content/70/2/197.long

6. Ferrero D, Pogliani E, Rege-Cambrin G, Fava C, Mattioli G, Dellacasa C, et al. Complete Reversion of Imatinib-Induced Hepatotoxicity in Chronic Myeloid Leukemia Patients by Low-Intermediate Dose Corticosteroid. [Internet]. Blood, 2005;106(11):4856. Available from: http://www.bloodjournal. org/content/106/11/4856?sso-checked=true

7. Perini G, Santos F, Funke V, Ruiz J, Neto B, Hamerschlak N. Nilotinib post-liver transplantation for acute hepatic failure related to imatinib [Internet]. Leuk Res, 2009;33(12):234-5. Available from: https://www.sciencedirect.com/science/article/ abs/pii/S0145212609002884?via\%3Dihub 